\title{
Programmable Network Context Adaptation for Content Delivery Services
}

\author{
Zhaohong Lai ${ }^{1}$, Joachim Sokol ${ }^{2}$, Klaus-Peter Eckert ${ }^{3}$, Alex Galis ${ }^{1}$, Richard \\ Lewis', Chris Todd ${ }^{1}$ \\ 1 University College London, Department of Electronic \& \\ Electrical Engineering, Torrington Place, London WC1E \\ 7JE \\ 2 Siemens AG; Corporate Technology, CT IC 2, Otto-Hahn- \\ Ring 6,81730 München, Germany. \\ 3 FhI FOKUS, Kaiserin-Augusta-Allee 31 D-10589 Berlin, \\ Germany
}

\begin{abstract}
This paper proposes an active/programmable-network-based Service Adaptation Manager (SAM) design to effectively deliver reliable and rich services for multimedia Content Delivery Networks (CDN). It shows that the CDN's service domain comprises two planes: Application Service plane and Network Service plane. The goal of the SAM is to manage the dynamics of the boundaries between these two planes for multimedia CDN (mCDN). The SAM includes three major parts: Service Context Adaptor, Service Adaptation Broker and Network Context Adaptor. A novel algorithm has been proposed to design the Service Context Adaptor. This allows different subsets of service context to be adaptively integrated in order to select the most suitable adaptation modules (such as VPN, QoS or the Optimal Route Service) for the delivered content. The proposed Network Context Adaptor algorithm provides an effective solution for detecting the network topology structure. Also, this paper proposes the design of an adaptive Optimal Route Service for CDNs.
\end{abstract}

\section{Introduction}

The advent of content distribution networks (CDN) has significantly improved Internet service performance and has a dramatic impact on the networking industry [3]. However, the traditional vendor-dependent or proprietary CDNs are facing the difficult challenge of supporting new services to customers [1][2] such as personalised services, increased security, flexible pricing etc. The fundamental reason for these difficulties is the strict separation between service layer and other CDN layers. A typical example is that the underlying network layer still only

Please use the following format when citing this chapter:

Lai, Z., Sokol, J., Eckert, K.-P., Galis, A., Lewis, R. and Todd, C., 2007, in IIIP International lederation for Information Processing, Volume 229, Network Control and Engineering for QoS, Security, and Mobility, IV, ed. Gaiti, D., (Boston: Springer), pp. 243-255. 
supports single class best-effort service, which cannot satisfy the rich services from the higher layer. As a new generation CDN design, the mCDN project adopts a multi-layer approach as the basis of its architecture, which mainly includes service layer, content distribution layer and network layer. The inter-layer design philosophy provides open interfaces between the different layers. To tackle the boundaries between service layer and network layer, as stated in [4], requires internetworking layers to minimise the gap between the service layer and network layer. This paper proposes an active-network-based Service Adaptation Manager design to effectively deliver reliable and rich services for multimedia CDNs [24] (multimedia Content Discovery and Delivery Network project). Firstly, this paper reviews the service deployment problems in traditional network-centric service environments. Then, it analyses the drawbacks of application-centric solutions in service adaptation development. After that, it presents a service-oriented view as the trend for the future service deployment. Based on this, the CDN's service domain is divided into two planes: Application Service plane and Network Service plane. The aim of the Service Adaptation Manager is to provide the required co-ordination between service layer and network layer for mCDN. It includes three major parts: Service Context Adaptor, Service Adaptation Broker and Network Context Adaptor. A novel algorithm is proposed as a key element of the design of the Service Context Adaptor. This allows different subsets of service context to be adaptively integrated in order to select the most suitable adaptation modules (such as VPN, QoS or the Optimal Route Service) for any delivered content. Also, the proposed algorithm for the Network Context Adaptor provides effective means to determine the network topology structure. Finally, how to develop the adaptive Optimal Route Service has been examined.

\section{Review in Service Adaptation}

\subsection{Services adaptation in the traditional network-centric network environment}

In traditional network design, services are considered as the one part of the network layer. In [6], Gbaguidi $e$ al referred this type of service deployment design as the network-centric based solution. In this framework, services are tied to specific technologies and rely on a dedicated signalling system. For example, the SS7 $7^{1}$ TUP $^{2}$ based switch can only support voice calls while video calls require an ISDN upgrade for every switch. Similarly, only the X.25 or Frame Relay networks support data services. It is clear that, in such an environment, a new service deployment task will be almost entirely implemented within the network and will take years to be completed. Therefore, new service adaptation only can be achieved by relying on the third-party vendors to replace/upgrade modules or install completely new switches.

1 - Signaling System No.7

2 - Telephone User Part 


\subsection{Service adaptation in the IP-based network environment}

In IP-based network environments, due to the clear separation of service layer and network layers, customers can develop their own services [4]. Typically, web services have been dramatically developed. Fundamentally, IP network is able to support rapidly growing services as IP is an open and connectionless layer 3 protocol. But, as the layer 2-4 protocols are unaware of the service requirements, the existing network treats all packets with the same single 'best effort' class [5]. As a result, this leads to many studies on service adaptation between the service layer and network layer.

In [4], Shenkef et al proposed the application taxonomy as the basis of implementing the service models. The fundamental philosophy of this scheme is that applications are regarded as central to the service structure. Therefore, if one application has been identified, its service type can be recognised and then service adaptation decisions can be made. This work divided network applications into two categories: elastic and real time application. The detailed application taxonomy paradigm is shown in Fig. 1. Based on this application taxonomy, service adaptation can be implemented to some extent. But, there exist obvious drawbacks in this solution such as the narrow service definition. Also, this solution does not fully consider the gap between service layer and network layers.

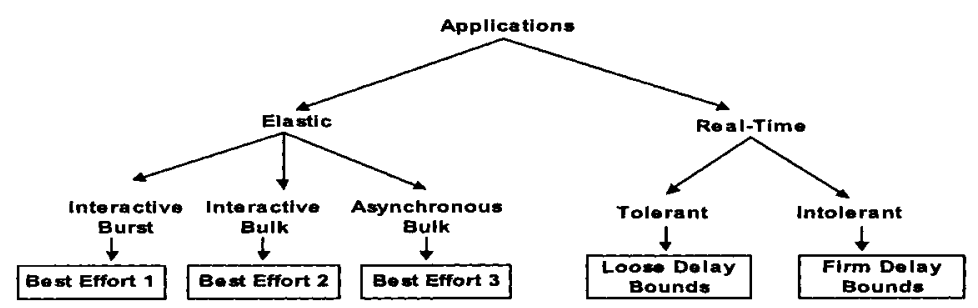

Fig. 1. Application Taxonomy- application classes, associated service offerings.

Compared with [4], Sun et al presented a much broader conceptual view on service adaptation in [7]. In their view, the input for adaptation is divided into two types: context-independent and context-dependent. The context-awareness based information is regarded as the implicit input, which leads to the additional elements for service adaptation. Also, a model to construct the adaptive services has been proposed. This model includes three methods: application transparent, application omniscient and application aware methods as shown below: 


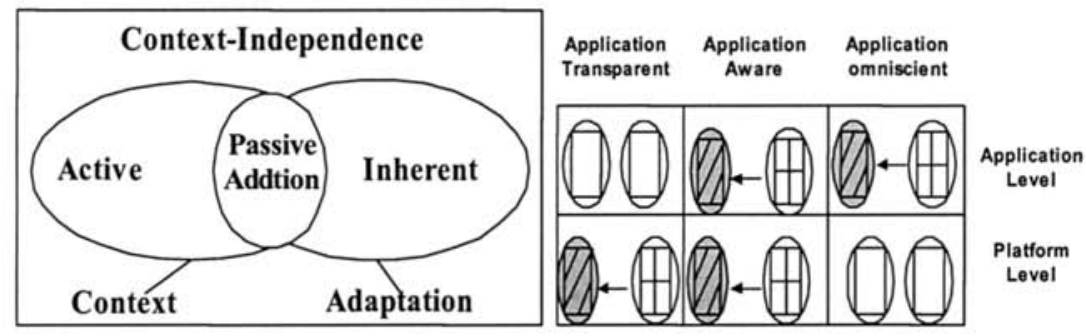

Fig. 2. Context-awareness and Adaptation (Left) and Classification of Adaptation model (Right) [7]

\subsection{Service adaptation realisation with active network approach}

In [7], based on context-awareness from multiple levels, implicit inputs are taken into account; the adaptation ability is therefore greatly improved. However, the proposed realisation model lacks an analysis of how applications can seamlessly interact with the network layer after they have obtained contextual information from platform level. To deal with issue, the active-network based intelligent solution has been recently popularised in research. As stated in [9][10][11][8], active network provides an innovative solution to open up the traditional closed networks so as to adaptively/intelligently deploy new services. In active network, the Execution Environment (EE) terminology refers to the runtime environment while the active code is called the Active Application (AA) terminology [22]. A wide range of EE and AA designs have been presented [14][15][16][17]. From the service adaptation view, in the Libra project design [10], Steenkiste et al proposed an elegant model to map into EE and AA, which includes two modules: the base service modules and customisation code module. The base service implements shared functionalities, while the customisation code module allows users to "fine tune" the service. Thus, service composition allows the development of new services by combining existing components from the base service module while the service adaptation functionality is implemented by customisation code module. This solution greatly reduces redundancy for deploying new services and effectively improves service adaptation scalability with a lightweight scheme in active network.

\section{Service-Oriented View in Content Delivery Network}

As discussed above, from the network-centric approach to the existing active network based solution, the service adaptability and scalability has been significantly improved. This evolution reflects the future trend for service adaptation that is moving to the service-oriented architecture design as shown below. 


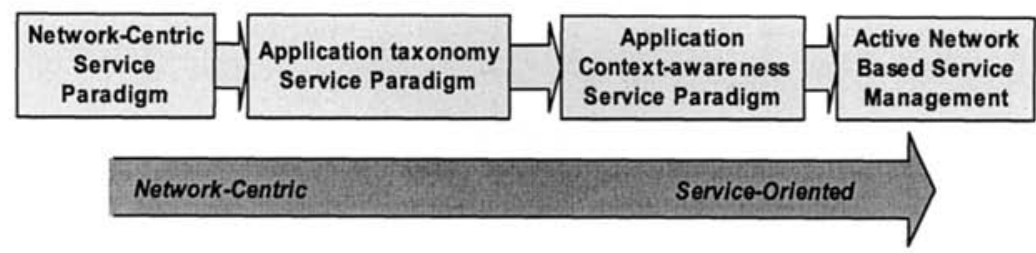

Fig. 3. Service Adaptation Evolution Steps

From the service-oriented point view, Jones defines service as a discreet domain of control that contains a collection of tasks to achieve related goals [24]. Specifically, this domain can be divided into two planes: network service plane (NSP) and application service plane (ASP) as shown in Fig. 4. In the Content Delivery Network context, the Network Service Plane mainly includes the Content Distribution Server (CDS), Metadata Distribution Server(MDS), Edge Servers(EDS) and other ISPs as shown below. The network service provider firstly provides the necessary physical network resource to ASPs like network nodes and its links and bandwidth etc. Secondly, NSPs also offer dedicated and specialised devices for ASPs such as cache machines, leased lines between CDS and EDS etc. The service domain in the application service provider mainly comprises content service provider, end users and other valued-added services etc.

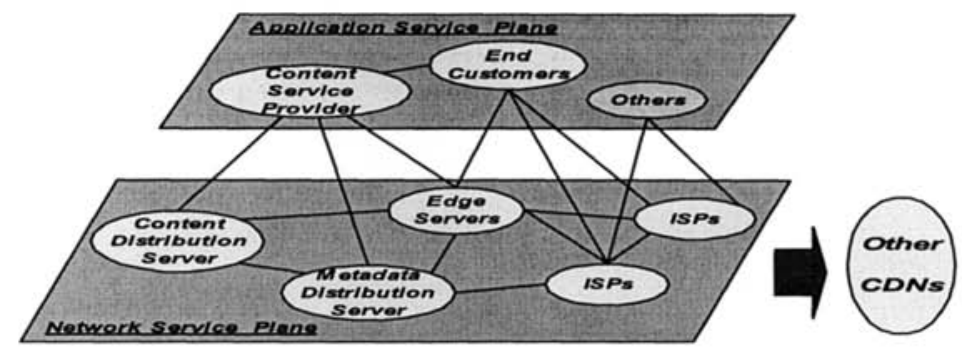

Fig. 4. Two service planes in service-oriented $\mathrm{CDN}$

In such a two-plane service domain, it is apparent if the programmable platform can be deployed between ASPs and NSPs, the goal of CDN's service adaptability, availability and scalability can be effectively achieved. However, the service adaptation tasks will not be able to effectively perform without the support from other service context-awareness components. As discussed in [7], the implicit information derived from context-awareness is the very important data input for service adaptation. In the ASP plane, the scope of the required contextual knowledge comprises user, location, device, content, application and other service context. In $\mathrm{mCDN}$ project design, two components ${ }^{3}$ have been developed to address these

${ }^{3}$ The details of the $\mathrm{mCDN}$ project architecture are at [23] 
issues: Personalization and Profiling System (PPS) and Internet Media Guide (IMG). The Personalization and Profiling Service PPS is the central component for CDN personalization issues. PPS is capable to deal with personalization and profiles within arbitrary scenarios. An Internet Media Guide (IMG) is a set of metadata describing the features of multimedia content. For example, metadata may consist of the URI, title, airtime, bandwidth requirements, file size, text summary, genre, and access restrictions. PPS and IMG provide the rich context-aware adaptation knowledge for CDN. In line with ASP, the network-aware Service Adaptation Manager is designed in NSP plane as discussed in next section.

\section{Service Adaptation Manager Design in CDN}

\subsection{Service Context Adaptor}

The Service Context Adaptor (SCA) is the core part for the Service Adaptation Manager (SAM) design. The SCA aims to effectively and adaptively utilise service context in order to construct service adaptation modules. The service context set is the chain from diverse aspects, which could be either explicit or implicit information from the ASP plane in CDNs. To effectively manage various service context data, the policy for constructing adaptation module needs to be methodically predefined. Based on this policy, the SCA is able to construct different adaptation modules. The example in Fig. 5 shows that the service context space has been divided into three subsets. The service adaptation module is varied according to different subset combinations, e.g. the Optimal Route Service module is consisted by the subset 2 and 3. Some elements are shared by different service adaptation module. For example, the 'Billing' context is part of both VPN and QoS service.

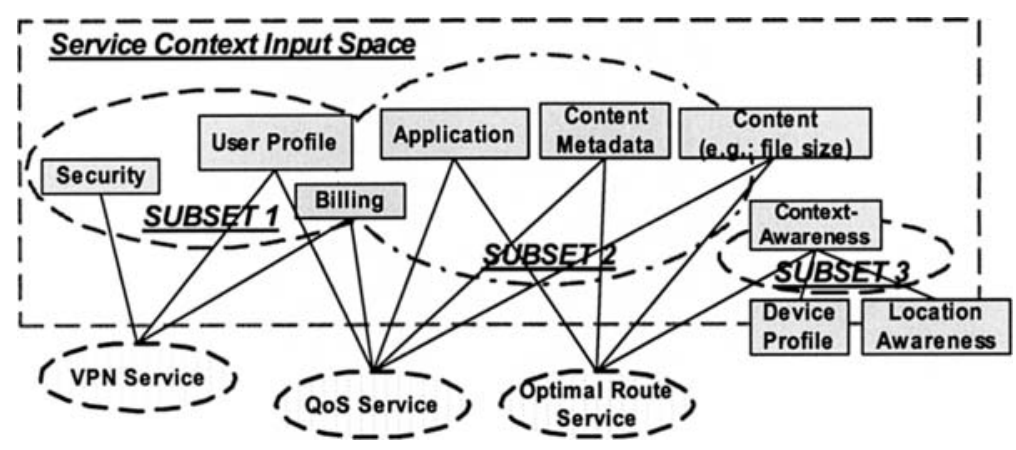

Fig. 5. The flexible Service Context Adaptor 
Here, the SCA algorithm is defined as follows. If the service context space is denoted as $S$, then the new service adaptation module $S_{m}$ is defined as:

$$
\left(\forall e_{1}, e_{2} \ldots e_{i} \in S\right)\left(\exists e_{1} . . e_{n} \in S_{i}\right)\left(\exists S_{m}=S_{1} \cup S_{5} \ldots \cup S_{i}\right)
$$

where $S_{i}$ is the subset of $S$ and the $e_{i}$ is element of service context subset. Normally, the $S_{i}$ is predefined with engineering approach. The condition of triggering a new service adaptation module is that every element's value in $S_{i}$ needs to reach the predefined threshold. It is difficult to give the exact value for each context element. But, we can define the level value for $e_{i}$. Also, the threshold policy set $S_{p}$ needs to be defined in advance. In this $S_{p}$, there are varied policies regarding to different element's value. For example, QoS service can be defined as different classes according to different billing types. When the predefined policy set $S_{p}$ for $S_{m}$ is satisfied the following equation, the service adaptation $S_{m . v}$ has real value and then $S_{m}$ will be triggered by active codes:

$$
\left(\forall e_{1}, e_{2} \ldots e_{i} \in S_{m}\right)\left(\exists\left(r_{e .1} \& r_{e .2} . . \& r_{e . i}\right) \in S_{p}\right) \Rightarrow\left(S_{m . v} \notin \Phi\right)
$$

here, $r_{e . i}$ is the $e_{i}$ 's rating value and $S_{m, v}$ is the flag for each $S_{m}$. There are different rating values for $e_{i}$. For example, if there are four bits representing billing's classes, its rating value can range from ' 0000 ' to ' 1111 ' for total sixteen types. The modular sum of rating value determines the module type of service adaptation. Based on (1), (2) and predefined policies, the Service Context Adaptor therefore can adaptively choose which service adaptation module should be triggered.

\subsection{Service Adaptation Broker based on DINA Platform}

In the $\mathrm{mCDN}$ project, the DINA ${ }^{4}$ active platform has been chosen for the adaptation work. A full and detailed review of programmable networks technology is described in [22]. The Service Adaptation Broker has been developed so as to deliver the service adaptation module with the 'on-the-fly' manner for Service Adaptation Manager. As a more advanced variant of $\mathrm{ABLE}^{5}$, DINA has a modular software architecture that enables deployment, control, and management of active services (sometimes called sessions or active sessions) over networks entities such as routers, WLAN access point, media gateways, and servers that support such services in IPbased networks. Its platform independent interfaces can be used by the active services in order to manage, control, retrieve information or perform other operations in the local node. DINA active platform consists of an Active Engine and a Forwarding Element, namely router, WLAN access point, media gateway, etc. (sec Fig.6).

\footnotetext{
${ }^{4} \mathrm{http} / / /$ context.upc.es/principal_licence.htm

${ }^{5} \mathrm{http} / / \mathrm{www} . \mathrm{cs}$.bell-labs.com/who/ABLE/
} 


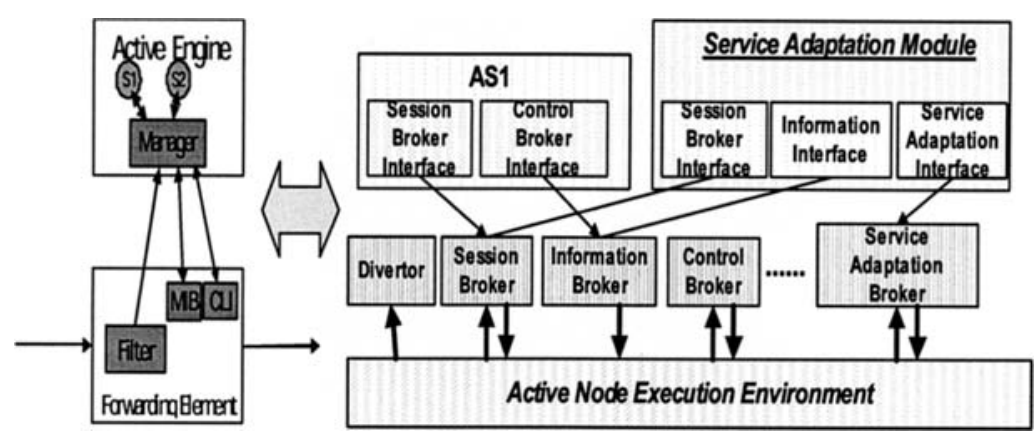

Fig. 6. Active Platform Environment

The modular design of DINA allows the different components to be logically separated. In particular the Active Engine and the Forwarding Element can be either physically separated or co-located at the same machine. This kind of architecture enables DINA to support platforms from different vendors using almost an identical software components implementation. The brokers are modules that give active services the capability to utilize host information and resources and perform operations in the local environment:

1. The Session Broker is the core on the active node. It receives and parses active packets, handles and manages existing services, and it distributes active packets according to requests of the services.

2. The Information Broker Interface provides services to retrieve information such as MIB database of the active host.

3. The Control Broker Interface enables active services to control and configure the active node.

The Service Adaptation Broker is developed as the modular platform that provides open interfaces to specific service adaptation modules such as VPN, QoS and Optimal Route Service modules.

\subsection{The objective of Service Adaptation Manager in CDN}

Service Adaptation Manager (SAM-Fig.7) is designed to seamlessly manage Service Context Adaptor, Service Adaptation Broker, Information Broker, Network Context Adaptor and Service Adaptation Modules. Its goal is to improve service availability and reliability with an optimal and adaptive approach in the changing CDN environment. Firstly, SAM needs to provide a seamless interface between ASP and NSP service plane as the CDN network layer can be aware of service requirement. Apart from that, as stated in section 4.1, the SCA is the core part of service adaptation decision-making. After referencing to predefined policies, it will select which adaptation module will be triggered and with which level, e.g., QoS service with different levels. Moreover, the network-level context will be taken into account by SAM, which is implemented by the Network Context Adaptor. 


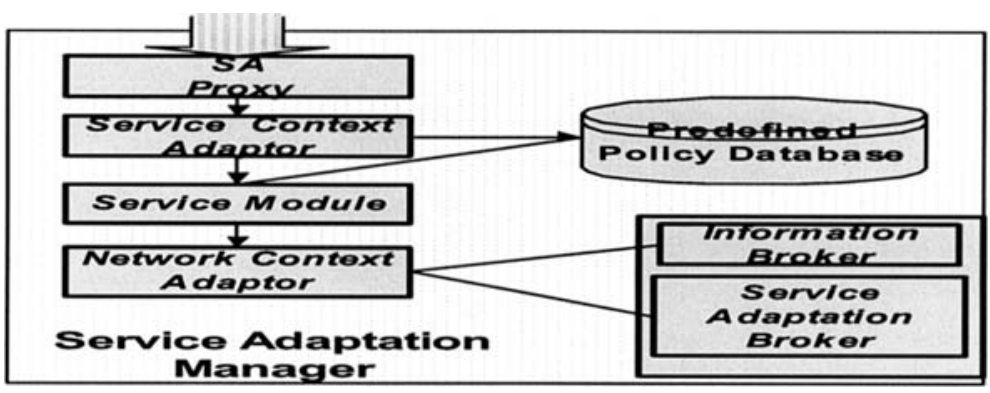

Fig. 7. Service Adaptation Manager

The network-aware service adaptation is a bottom-up solution, taking a passive approach. Typically, when the default network link is congested or broken, the timesensitive service needs to adapt to this change. Many studies propose network-aware service adaptation [18][19][20][21] based on the application and network transport level, but the limited functionalities can be achieved. Using the built-in functionalities of DINA, the network-level contextual information can be retrieved by SAM, like MIB data from Information Broker. In the next section, it examines how to design the Optimal Route Adaptation Service by SAM.

\section{Optimal Route Adaptation Service Module}

\subsection{The Content Service Context for ORS design}

The Optimal Route Adaptation Service (ORS) component design follows a serviceoriented approach to allocate network resource for CDN's applications. This is due to the fact that as existing routing protocols like OSPF do not consider service information. As a result, if one unmatched link has been chosen for one multimedia content delivery, content quality and QoS can be expected to severely degrade. The ORS is a typical adaptive service design, which considers service context information from both the ASP plane and NSP plane.

As discussed in 4.1, the type of service module is defined by Service Context Adaptor. The nature of the content is required to define the ORS module. Specifically, the input for Service Context Adaptor needs content metadata information such as content service type, bandwidth requirement and the size of content. One example of content service type is shown in the Table 1. According to this table, ORS will verify the predefined policy database and make different decisions, e.g. the flow reservation required for the '0101' type. 


\begin{tabular}{|l|l|}
\hline $0001 \sim$ Best effort & $0101 \sim$ mission critical (e.g.; medical video) \\
$0010 \sim$ voice content & $0111 \sim$ dynamic real-time content (financial stock data) \\
$0011 \sim$ video content & $1000 \sim$ emergency content \\
$0100 \sim$ voice \& video & \\
\hline
\end{tabular}

Table 1: Content Service Types

After the ORS module has been chosen by the SCA, the SAM will trigger the ORS internal functionality. In particular, ORS will interact with the Network Context Adaptor as to obtain the best route for the given content.

\subsection{The Network Context Adaptor Design for ORS}

Network Context Adaptor (NCA) is another key part of the design for Service Adaptation Manager. It is designed to obtain the network-level context-awareness information in dynamically changing network environments. Based on the networklevel contextual information, ORS can adaptively choose the optimal route for the given content. The information mainly includes the network topology, the available bandwidth and the link delay contextual information. Of these network metrics, it is vital to obtain the network topology picture so that ORS can know how many links/routes are available for the same application in a given network environment. Most network metrics can be directly obtained from reading the MIB database such as the physical bandwidth, the available bandwidth and the link delay etc. However, it is difficult to obtain and maintain the accurate network topology in a large-scale network, e.g. AS-level [13]. Computation of the complete network topology is a core part for the Network Context Adaptor design. In this paper, we only focus on how to obtain the network topology knowledge in one sub-AS area as ORS and SAM are designed as a distributed system.

\section{- The algorithm to obtain the Network Topology in ORS}

In one sub-AS area, the network topology can be deduced by the following (3) and (4) equations. If there are $\mathrm{N}$ nodes in one network topology, the total number of possible links $\varphi$ is given as:

$$
\varphi=2 * C_{n}^{2}
$$

where $n$ is equal to $N$. The number of possible routes $\Theta$ between any two nodes is given as:

$$
\Theta=\sum_{r=0}^{n} \mathrm{P}^{r}
$$


where $\mathrm{n}=\mathrm{N}-2$. This is can be examined by the following network scenario (Fig.8). In this scenario, one sub-AS area compromises 5 nodes and four stream contents will be delivered from A to A'.

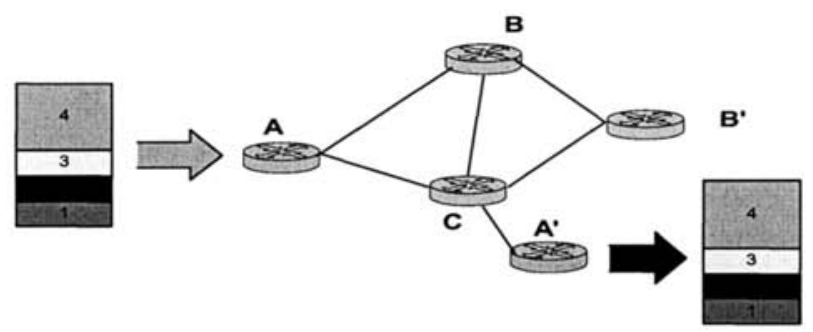

Fig. 8. One five-node's network topology algorithm in ORS

Applying the above equations, from node $\mathrm{A}$ to $\mathrm{A}^{\prime}$, theoretically, the number of routes is: $\Theta=\sum_{r=0}^{3} \mathrm{P}_{3}^{r}=P_{3}^{0}+P_{3}^{1}+P_{3}^{2}+P_{3}^{3}=16$, as $\mathrm{n}$ is equal to 3 (N-2). The total link number is: $\varphi=2 * C_{5}^{2}=20$.

Physically, there exist 12 links in this network shown below:

(1) $\mathrm{AB}, \mathrm{AC}, \mathrm{BC}, \mathrm{BB}$ ', $\mathrm{CB}^{\prime}, \mathrm{CA}^{\prime}, \mathrm{BA}, \mathrm{CA}, \mathrm{CB}, \mathrm{B}^{\prime} \mathrm{B}, \mathrm{B}^{\prime} \mathrm{C}, \mathrm{A}^{\prime} \mathrm{C}$;

Then, the total route number from $A$ to $A^{\prime}$ can be obtained via the following steps:

(2) $P^{0}: \mathrm{AA}^{\prime}=$ boolean: $\{0\}$, as the link $\mathrm{AA}^{\prime}$ can not be found in (1);

3

(3) $P^{1}: \mathrm{B}, \mathrm{B}^{\prime}, \mathrm{C}=\left\{\left\{\mathrm{AB}, \mathrm{BA}^{\prime}\right\},\left\{\mathrm{AB}^{\prime}, \mathrm{B}^{\prime} \mathrm{A}^{\prime}\right\},\left\{\mathrm{AC}^{\prime}, \mathrm{CA}^{\prime}\right\}\right\}=$ boolean: \{

$\{0,0\},\{0,0\},\{1,1\}\}=\{0,0,1\}$. Thus, only the link ACA' is true.

(4) $P_{3}^{2}: \mathrm{BB}^{\prime}, \mathrm{BC}, \mathrm{B}^{\prime} \mathrm{B}, \mathrm{B}^{\prime} \mathrm{C}, \mathrm{CB}, \mathrm{CB}^{\prime}=$ boolean: $\{0,1,0,0,0,0\} ; \rightarrow$ the link $\mathrm{ABCA}^{\prime}$ ' is true.

(5) $P^{3}: \mathrm{BB} \mathrm{B}^{\prime} \mathrm{C}, \mathrm{BCB}$ ', $\mathrm{B}^{\prime} \mathrm{BC}, \mathrm{B}^{\prime} \mathrm{CB}, \mathrm{CBB}, \mathrm{CB}{ }^{\prime} \mathrm{B}=$ boolean: $\{1,0,0,0,0,0\} ; \quad \rightarrow$ 3

the link $\mathrm{ABB}^{\prime} \mathrm{C}$ is true;

Therefore, there are 3 links that are available to deliver the content from node $\mathrm{A}$ to $A^{\prime}$ in this scenario. Once ORS has obtained the network topological context information, the SAM will ask the Information Broker to retrieve the minimum available BW for each available link. Finally, ORS can make decisions to optimise content delivery while also maximising network resource utilisation. 


\section{Conclusion}

This paper presents the design of a Service Adaptation Manager (SAM) based on programmable network technology, which provides a service adaptation capability for CDNs. It reviews the main drawbacks of existing solutions for service adaptation and it presents the two-plane service domain approach: Application Service Plane and Network Service Plane. It proposes the novel Service Context Adaptor algorithms for the provisioning of flexible and adaptive service adaptation and the computation of the network topology. Also, this paper particularly addresses how to design adaptive Optimal Route Service for CDNs.

\section{Acknowledgements}

This paper describes work partially undertaken in the context of the mCDN (FP6507993) - Information Society Technologies project, which is partially funded by the Commission of the European Union. We thank mCDN consortium for their good cooperation in the project.

\section{Reference}

1. Hosanagar, K.; et al, Optimal pricing of content delivery network (CDN) services, System Sciences, 2004. Proceedings of the 37th Annual Hawaii International Conference on,5-8 Jan. 2004 Page(s):205 - 214

2. Lazar, I.; Terrill, W., Exploring content delivery networking, IT Professional Volume 3, Issue 4, July-Aug. 2001 Page(s):47 - 49

3. Vakali, A.; Pallis, G., Content delivery networks: status and trends, Internet Computing, IEEE,Volume 7, Issue 6, Nov.-Dec. 2003 Page(s):68 - 74

4. Shenker, S.; Clark, D.D.; Lixia Zhang, Services or infrastructure: why we need a network service model, Community Networking Integrated Multimedia Services to the Home, 1994., Proceedings of the 1 st International Workshop on 13-14 July 1994 Page(s):145 - 149

5. Fuin, D.; et al, A novel approach to quality of service in active networks, Local Computer Networks, 2004. 29th Annual IEEE International Conference on, 16-18 Nov. 2004 Page(s):760 - 767

6. Gbaguidi, C., et al., A programmable architecture for the provision of hybrid services, Communications Magazine, IEEE,Volume 37, Issue 7, July 1999 Page(s): $110-116$

7. Title 15: Sun, J.-Z.; Sauvola, J., Towards a conceptual model for contextaware adaptive services, PDCAT'2003. Proceedings of the Fourth International Conference on 27-29 Aug. 2003 Page(s):90 - 94 
8. Hirschfeld, R.; Kawamura, K., Dynamic service adaptation, Distributed Computing Systems Workshops, Proceedings. 24th International Conference on 2004 Page(s):290 - 297

9. I. W. Marshall, et al, Active Management of multi-service networks, BT Labs, Adastral Park, Martlesham Heath Ipswich IPS 3RE

10. Steenkiste, P., et al, An active networking approach to service customization, DARPA Active NEtworks Conference and Exposition,Proceedings, 2930 May 2002 Page(s):305 - 318

11. Su Sen, et al, An intelligent network architecture based on active network technology, Communication Technology Proceedings, WCC - ICCT 2000. International Conference on Volume 2, Page(s): 1081 - 1086 vol.2

12. Jones, S., Toward an acceptable definition of service [service-oriented architecture], Software, IEEE,Vol22, Issue 3, May-Jun 2005 Page(s):87 93.

13. Aho, A.V.; Lee, D.; "Hierarchical networks and the LSA N-squared problem in OSPF routing",GLOBECOM '00. IEEE, 397 - 404 vol.1

14. D. Decasper, Z. Dittia, G. Parulkar, and B. Plattner. Router Plugins: A Software Architecture for Next Generation Routers. In Proceedings of the ACM SIGCOMM '98 conference, pages 229-253

15. D. J. Wetherall et al. ANTS:A toolkit for building and dynamically deploying network protocols. In IEEE OPENARCH '98, April 1998.

16. D. S. Alexander, et al, The SwitchWare active network architecture. IEEE Network, May/June 1998

17. S. Bhattacharjee, et al. An Architecture for Active Networking. In High Performance Networking (HPN'97), NY, April 1997

18. Bolliger, J., et al, 1999, "Bandwidth modelling for network-aware applications",INFOCOM '99. Eighteenth Annual Joint Conference of the IEEE Computer and Communications Societies Proceedings. IEEE, Volume 3, 21-25 March 1999 Page(s):1300 - 1309 vol.3

19. Khan, J.I.; Qiong Gu,2001,'Network aware video transcoding for symbiotic rate adaptation on interactive transport", NCA 2001. IEEE International Symposium on 8-10 Oct. 2001 Page(s):201 - 212

20. Bolliger, J.; Gross, T.R.; 2001, "Bandwidth monitoring for network-aware applications", HPDC 2001. Proceedings. 10th IEEE International Symposium on 7-9 Aug. 2001 Page(s):241 - 251

21. Bolliger, J.; Gross, T.; 1998, "A framework based approach to the development of network aware applications", Software Engineering, IEEE Transactions on Volume 24, Issue 5, May 1998 page(s):376 - 390

22. Galis, A., et al," Programmable Networks for IP Service Deployment" ISBN 1-58053-745-6; pp450, June 2004; Artech House Books

23. $\mathrm{mCDN}$ Project public website, http://www.comtec.e-technik.unikassel.de/content/projects/mcdn/pub/pages/mCDN_PublicDeliverables.html.

24. Jones, S., Toward an acceptable definition of service [service-oriented architecture], Software,IEEE,Vol.22, Issue 3, May-June 2005,Page(s):87-9 